\title{
RIESGOS EN EL TELETRABAJO EN DOCENTES DE EDUCACIÓN BÁSICA
}

\author{
RISKS IN TELEWORKING IN BASIC EDUCATION TEACHERS
}

\author{
Mauricio López-Acosta* \\ Allán Chacara-Montes ${ }^{* *}$ \\ José Manuel Velarde-Cantú ${ }^{* * *}$ \\ Ernesto Ramírez-Cárdenas ${ }^{* * *}$ \\ Grace Erandy Báez-Hernández ${ }^{* * * *}$
}

\begin{abstract}
Resumen: A principios del año 2020 a causa de la pandemia por el COVID-19 miles de docentes en México cambiaron la forma tradicional de impartir sus clases a realizarlas de manera remota, actividad denominada home office o teletrabajo, un cambio de modalidad de trabajo súbito, pues no cambió solamente el canal de comunicación, sino que también se modificaron las condiciones de trabajo, desde un salón de clases a algún puesto dentro del hogar. Este proyecto tuvo como objetivo conocer las experiencias de los docentes e identificar los riesgos ergonómicos a los que estuvieron expuestos con esta modalidad. Los sujetos de estudio fueron los docentes de educación básica que residen en la región del noroeste de México. Para realizar la investigación, se aplicó un cuestionario, adaptación de uno realizado por la Universidad de Concepción y la Sociedad Chilena de Ergonomía denominado "entorno ergonómico del teletrabajo en situación de pandemia", y se colectaron las respuestas por medio de formularios de Google. El nivel de confianza fue de un 90\%. Entre los resultados más significativos está que un $84,11 \%$ no había realizado teletrabajo antes de la pandemia, un $48,37 \%$ no recibieron instrucciones de cómo realizar las tareas de la mejor manera, un 79,42\% no tuvo entrenamiento y/o capacitación en ergonomía, un 71,11\% realizó menor actividad física durante el teletrabajo en comparación con el trabajo de clases normal, un $58,11 \%$ no contaba con escritorio, silla ajustable o reposapiés, un $45,81 \%$ no contaba con un lugar fijo de trabajo, y un $38,63 \%$ consideraba su lugar de trabajo como ruidoso. Los resultados también muestran que la mayoría, tuvieron alguna molestia o la necesidad de adaptar su lugar de trabajo.
\end{abstract}

Palabras clave: Ergonomía, riesgos, teletrabajo, home office.

Abstract: At the beginning of the year 2020, due to the COVID-19 pandemic, thousands of teachers in Mexico changed the traditional way of teaching their classes to remote teaching, an activity called home office or telework, a sudden change of work modality, since not only the communication channel changed, but also the working conditions were modified, from a classroom to a position at home. The

\footnotetext{
"Instituto Tecnológico de Sonora. Navojoa, México. Correo electrónico: mauricio.lopez@itson.edu.mx. Orcid: https://orcid.org/oooo-0003-3728-9576. Autor de correspondencia.

***Instituto Tecnológico de Sonora. Navojoa, México. Correo electrónico: allan.chacara@itson.edu.mx. Orcid: https://orcid.org/oooo-0002-0567-0017

***Instituto Tecnológico de Sonora. Navojoa, México. Correo electrónico: jose.velarde@itson.edu.mx. Orcid: https://orcid.org/oooo-0002-1697-8551

*****Instituto Tecnológico de Sonora. Obregón, México. Correo electrónico: eramirez@itson.edu.mx. Orcid: https://orcid.org/o0oo-0002-5248-724X

*****Instituto Tecnológico Superior de Guasave. Guasave, México. Correo electrónico: gracebaezh@gmail.com. Orcid: https://orcid.org/oooo-0oo3-1580-2199
} 
objective of this project is to learn about the teachers' experiences and identify the ergonomic risks to which they were exposed with this modality. The subjects of the study were elementary school teachers residing in the northwestern region of Mexico. To carry out the research a questionnaire was applied, adapted from one made by the University of Concepción and SOCHERGO called "ergonomic environment of telework in pandemic situation", the answers were collected by means of Google forms. The confidence level is $90 \%$. Among the most significant results is that $84.11 \%$ had not performed telework before the pandemic, $48.37 \%$ did not receive instructions on how to perform the tasks in the best way, $79.42 \%$ have no training and/or training in ergonomics, $71.11 \%$ have less physical activity compared teleworking with normal classroom work, $58.11 \%$ do not have a desk, adjustable chair or footrest, $45.81 \%$ do not have a fixed place to work, $38.63 \%$ consider their workplace as noisy. The results also show that the majority have had some discomfort or have the need to adapt their workplace.

Keywords: Ergonomics, risk, telework, home office.

Recepción: 27.10.2021 / Revisión: 05.11.2021 / Aceptación: 07.12.2021

\section{Introducción}

Estados Unidos es uno de los pioneros en el teletrabajo, para apoyar esta idea, en el año 1988 California ya había establecido un programa piloto de teletrabajo. Sin embargo, es hasta 2010 que el teletrabajo es definido por la ley 111 en diciembre en este país (EU congress, 2010, citado por Rodríguez-Moreno, 2017), y es en ese mismo año que se dicta la Ley de Mejora del Teletrabajo o Telework Enhancement Act en inglés (Álvarez, 2018, octubre). Actualmente, como señala Rodríguez-Moreno (2017), las disposiciones por parte del gobierno varían dependiendo del estado, las similitudes en las políticas son sobre horas de trabajo, localización, aspectos de seguridad, así como criterios de selección y terminación según señaló un estudio realizado en 35 estados del país.

En cuanto a la Unión Europea, en el año 2001 el Comité de Diálogo Social del Sector de las Telecomunicaciones publicó un documento con indicaciones que debían aplicarse al teletrabajo de las Telecomunicaciones en Europa. Para el 16 de julio de 2002 la Confederación Europea de Sindicatos, la Unión de Confederaciones de la Industria y de Empresarios de Europa, la Unión Europea del Artesanado y de la Pequeña y Mediana Empresa y el Centro Europeo de la Empresa Pública, firmaron el Acuerdo Marco Europeo sobre Teletrabajo, que en términos propios "busca modernizar la organización del trabajo" con el fin de aumentar la productividad y la competitividad, así como aumentar el nivel de seguridad y capacidad de adaptación a distintas formas de trabajo (Rodríguez-Mejía, 2007).

A pesar de que en América Latina se ha tratado de incluir el teletrabajo como una modalidad para brindar empleo, la estructuración de leyes y normas en la mayoría de los países no es suficiente y hace que sea muy difícil llevarlo a cabo de manera formal (Fernández-Tapia \& Bravo-Salazar, 2019). Por otra parte, Argentina se ha ubicado como el principal precursor del teletrabajo en América Latina (Osio-Havriluk, 2015), esto se evidencia en sus antecedentes, entre los más destacados: en 2003 se crea la Comisión de Teletrabajo correspondiente al Ministerio de Trabajo, Empleo y Seguridad Social (Ramírez, 2008, como 
cita Osio-Havriluk, 2015); en el año 2007 se promulga la Ley Régimen Jurídico del Teletrabajo. Además de iniciativas del sector privado para regular el teletrabajo, la más destacada es de la empresa Telecom, con sede en Buenos Aires, la cual desarrolló su propio manual para regular el teletrabajo (Osio-Havriluk, 2015).

Otro país que cuenta con reglamentación del teletrabajo por parte del gobierno es Colombia, el teletrabajo está regulado por la Ley 1221 del 2008 y el decreto 884 del 2012 que la reglamenta. Mientras que la Ley 1221 reconoce al teletrabajo como modalidad de trabajo, el decreto 884 especifica las condiciones laborales para entidades privadas y públicas, las obligaciones y derechos del empleador y teletrabajador (Barona-Betancourt, 2013).

En México, como menciona Valle-Mendoza (2018) el trabajo a distancia es una tendencia que se espera gane mayor importancia al pasar el tiempo. En México, se cuenta con un espacio en la Ley Federal del Trabajo en el título sexto bajo el nombre de "trabajos especiales" capítulo Xll "trabajo a domicilio" con varios artículos que trata de llevar un control de las actividades de trabajo en el hogar (Secretaría de Gobernación, 2019, 2 de julio), sin embargo, la definición que se da en el artículo 311 del trabajo en el hogar no es lo mismo que el teletrabajo o home office.

Lo anterior significa que el teletrabajo no se encuentra regulado de manera detallada en la legislación laboral existente, tan solo se convalida con el trabajo a domicilio, lo cual es distinto al término de teletrabajo, una de las principales diferencias entre los dos términos es que los servicios del trabajador a domicilio terminan en el momento de entregar el producto terminado; con el teletrabajador se mantiene una situación laboral estable, donde las actividades pueden variar en forma continua (Silva-Ambríz et al., 2013).

Con el paso del tiempo se ha tratado que aumente el nivel de aceptación del teletrabajo con diferentes estrategias, como la denominada "oficina en tu casa" que se llevó a cabo en la ciudad de México en 2017 (Organización Internacional del Trabajo [OIT], 2017). En cuanto a avances legales por parte del gobierno, la Comisión de Trabajo y Previsión Social en la Cámara de Diputados aprobó el dictamen que busca regular el teletrabajo en México, la cual ya había sido presentada por los senadores en el 2018, por lo cual se reformará el artículo 311 de la Ley Federal del Trabajo dando como resultado al capítulo Xll bis (Martínez, 2020). Con la reforma del artículo se aceptará por ley el término del teletrabajo y se agregarán especificaciones sobre distintos puntos del teletrabajo, modificaciones que aún están en proceso.

Actualmente, en marzo del año 2020 se decidió que no se regresaría a las aulas porque no se contaba con las condiciones necesarias contra el COVID-19 en México (Sector Salud, 2020) por lo que las clases se comenzaron a impartir de manera remota/virtual desde nivel básico hasta nivel superior lo que significó el cambio de la modalidad de trabajo de presencial a virtual para docentes y estudiantes de todos los niveles educativos, causando una modificación a las condiciones de trabajo para los educadores y estudiantes.

Como lo describen Cifre-Gallego y Alonso-Fabregat (2002), el teletrabajo trae consigo nuevas necesidades y demandas, así como riesgos a la salud. Los riesgos existentes en el teletrabajo a la salud se pueden relacionar con los que se presentan en los puestos de trabajo en oficinas, pues, ambas tareas se realizan con ayuda de tecnologías de la información y 
comunicación. Por otro lado, como menciona Mondelo et al. (2013) en las oficinas se presentan problemas musculoesqueléticos principalmente por la posición frente a la mesa de trabajo y el ordenador. Sumado a lo anterior, según Erika Villavicencio investigadora de la UNAM, menciona que solo 2 de cada 10 empresas en México están preparadas para aplicar la modalidad de teletrabajo a raíz de la emergencia sanitaria por el COVID- 19 (Miranda, 2020, 19 de marzo).

Ante este contexto se plantea la siguiente pregunta: ¿̇cuáles son los principales factores de riesgo ergonómico en la modalidad de teletrabajo en los que están expuestos los docentes de educación básica?

\section{Objetivo}

Conocer el entorno ergonómico en donde se desarrolla el teletrabajo actualmente en los docentes de nivel básico por medio de un instrumento en línea con el fin de obtener propuestas de mejora.

\section{Materiales y métodos}

\section{Participantes}

Se determinó como sujetos bajo estudio en la investigación a los docentes de educación básica (preescolar, primaria y secundaria) residentes en el noroeste de México.

\section{Procedimiento}

Para el cumplimiento de los objetivos planteados, se presenta a continuación el orden de los pasos a seguir.

Determinación de la muestra

1. Identificación del número total de la población de los sujetos bajo estudio por medio del anuario estadístico y geográfico por entidad federativa llevado a cabo por el INEGI en el 2019.

2. Cálculo de la muestra de proporción con población finita mediante la siguiente fórmula (Valdivieso-Taborga et al., 2011):

$$
n=\left\lceil N *\left(Z^{2}\right) * p * q\right\rceil /\left\lceil\left(e^{2}\right) *(N-1)+\left(Z^{2}\right) * p * q\right\rceil 3
$$

Diseño del instrumento

1. Adaptación del instrumento desarrollado por el Departamento de Ergonomía de la Universidad de Concepción y la Sociedad Chilena de Ergonomía (SOCHERGO), denominada "entorno ergonómico del teletrabajo en situación de pandemia" (2020) a un formato que permita la distribución y respuesta a distancia y de manera rápida.

Las preguntas se muestran agrupadas como se observa a continuación:

- Información general. 
- Situación laboral.

- Experiencia en el teletrabajo.

- Alimentación y actividad física.

- Condiciones para el desarrollo teletrabajo y aspectos posturales.

- Aspectos del ambiente.

- Aspectos de carga mental, exigencias laborales y horarios de trabajo.

- Teletrabajo y consecuencias.

2. Ajuste de las preguntas que lo requieran para que sean acordes a la cultura del sujeto de investigación y adición de preguntas en los espacios donde que se necesite mayor información.

En el proceso de adaptación se agregaron dos apartados denominados "situación laboral" y "teletrabajo y consecuencias", respectivamente. El primer punto se añadió para verificar si quien contesta pertenece al grupo de interés de la investigación y, el segundo punto, se añadió para que las personas encuestadas dieran respuesta de manera general sobre la aceptación hacia el teletrabajo.

Análisis de resultados

1. Se desglosaron los resultados obtenidos por medio del cuestionario, expresaron en porcentajes y se presentaron de manera gráfica.

2. Se identificaron los puntos que tuvieron mayor porcentaje con connotación negativa, por ejemplo: no cuenta con, no lo considera o cuenta con molestias.

Los materiales utilizados fueron el instrumento mencionado y las redes sociales para su difusión.

\section{Resultados y discusión}

Considerando una población finita de $\mathrm{N}=152.524$ y un nivel de confianza de un $90 \%$, se obtuvo una muestra de $\mathrm{n}=272$ sujetos, distribuidos como se observa en la figura 1 . 
Figura 1. Región de aplicación del instrumento.

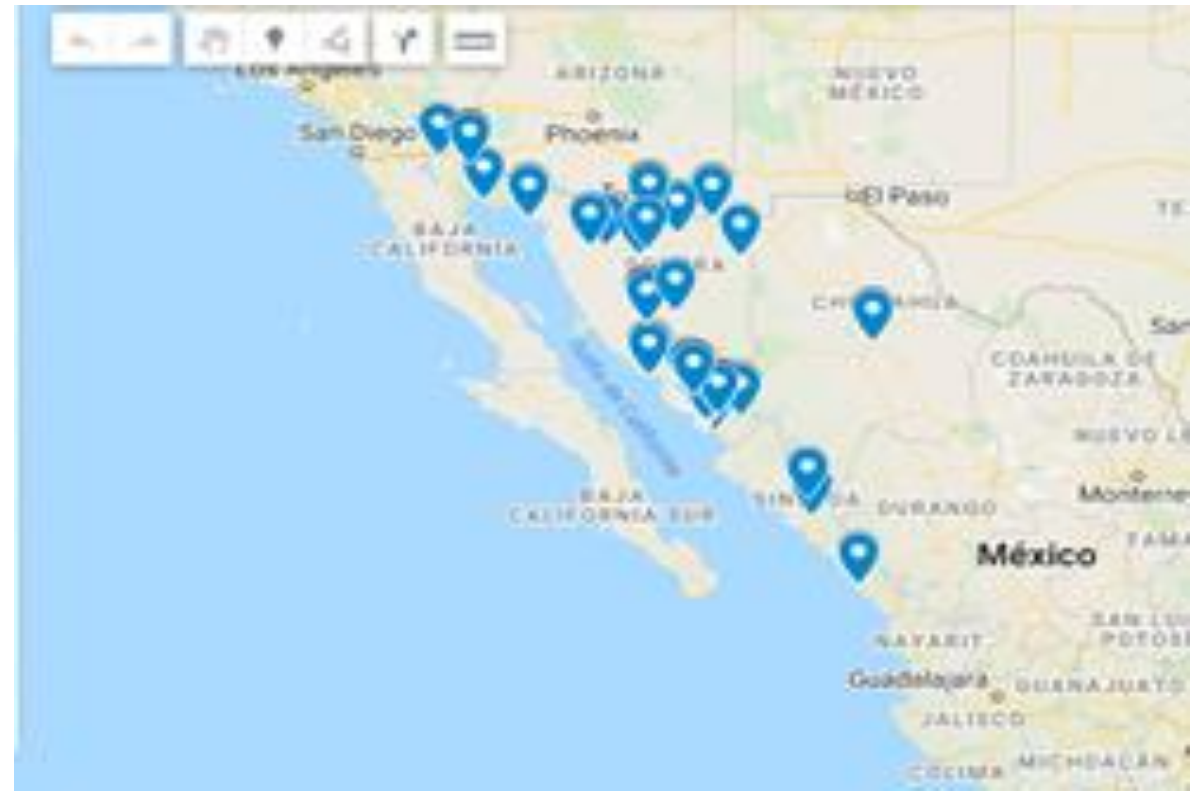

A continuación se presentan los resultados más relevantes:

1. Las encuestas contestadas por los docentes de educación básica fueron un total de 277.

2. Un $71,84 \%$ de las encuestas fueron contestadas por mujeres y el $28,15 \%$ por hombres.

3. El 53,79\% de las personas que participaron tienen entre 20-29 años, el segundo grupo con mayor porcentaje fue el de 30-39 años con un 21,66\%, el tercer grupo fue el de 40-49 años con un 14,07\%, el cuarto grupo fue el de 50-59 años con un 9,02\%, el quinto grupo fue el de 60-69 años con un 1,08\% y el último grupo fue el de >70 años con un $0,36 \%$.

4. El 47,29\% de la muestra está soltero, el 44,76\% de la muestra está casado/a, el 5,77\% de la muestra está en convivencia, el $0,72 \%$ de la muestra está viudo y por último el 1,44\% de la muestra está divorciado.

5. El 4,33\% vive solo, el 12,99\% vive con 2 personas, el 22,01\% vive con 3 personas, el 31,41\% vive con 4 personas y un 29,24\% vive con 5 o más personas.

6. El 64,98\% vive con adultos, el 22,02\% vive con adolescentes, el 6,13\% vive con niños de 6 a 10 años, el 2,16\% vive con niños de 3 a 5 años y el 4,69\% vive con bebés lactantes.

7. El 1,08\% tienen educación media, el o,36\% tienen estudios técnicos, el 54,87\% tienen estudios universitarios, el 34,29\% tienen maestría y el 9,38\% tienen doctorado.

8. El 32,85\% de la población vive en hogares sobre los 50 y 80 m2, el 27,07\% de la población vive en hogares sobre los 81 y 100 m2, el 20,93\% sobre los 101 y 140 m2 y el 19,13\% sobre más de $140 \mathrm{~m} 2$.

9. Un $43,32 \%$ tiene contrato a plazo fijo, un $45,84 \%$ tiene contrato indefinido, un $2,16 \%$ tiene trabajo independiente y un $8,66 \%$ es independiente y dependiente.

10. Un 80,14\% se encuentra en el sector educación básico-público y un 19,85\% se encuentra en el sector educación básico-privado. 
11. Un $15,88 \%$ habían realizado teletrabajo y un $84,12 \%$ de las personas no habían desarrollado teletrabajo antes del COVID-19.

12. Entre quienes desarrollaban teletrabajo previo al COVID-19, un $76 \%$ desarrollaban teletrabajo en casa y un 24\% desarrollaban teletrabajo en casa y/o en lugares públicos.

13. Sobre el $48,37 \%$ de las personas sin experiencia en teletrabajo, no recibieron instrucciones de cómo ejecutar las tareas de la mejor forma.

14. Las ventajas sobre el teletrabajo son, menores costos $67,15 \%$, mayor flexibilidad $57,04 \%$, mejor conciliación vida familiar y trabajo $28,52 \%$, el $9,39 \%$ indica tener la posibilidad de realizar teletrabajo aumenta su satisfacción laboral, 1,44\% otra y un 4,69 \% no encuentra ninguna ventaja.

15. Un 20,77\% tienen entrenamiento y/o capacitación formal en ergonomía y un 79,42\% no lo tienen. Las personas con capacitación en ergonomía indican mejor salud, productividad, calidad y mejores relaciones laborales.

16. Un $66,42 \%$ de las personas realizó ajustes en su forma de alimentación y el $33,57 \%$ no realizó ajustes en su forma de alimentación.

17. Un $44,76 \%$ de las personas manifiesta que ha aumentado su peso, un $38,98 \%$ se ha mantenido en su peso y un $16,24 \%$ ha disminuido su peso.

18. Un 40,07\% de las personas comen lo mismo en cantidad y calidad, un $22,38 \%$ comen más cantidad, pero más saludable, un 19,49\% comen más cantidad, pero menos saludable y un $18,05 \%$ comen menos y más saludable.

19. Un $49,09 \%$ no realiza actividad física en forma regular, un $28,15 \%$ realizan actividad física de 1 a 3 veces en la semana y un 22,74\% realizan actividad física 3 o más veces por semana.

20. Un $71,12 \%$ de personas tienen menor actividad física comparando el teletrabajo con el trabajo de oficina tradicional, un 14,08\% tienen la misma actividad física y un 14,80\% tienen mayor actividad física.

\section{Condiciones para el desarrollo del teletrabajo y aspectos posturales}

1. Sobre las tecnologías utilizadas, un $75,81 \%$ indica trabajar con notebook, un $88,09 \%$ con teléfono inteligente, un 19,49\% con pc fijo y un 19,13\% con tabletas.

2. Un $46,93 \%$ cuenta con un escritorio, un $22,74 \%$ tiene una silla ajustable, un $2,89 \%$ tiene reposapiés y un 50,18\% no tiene ninguno de los anteriores.

3. En el caso del notebook, un $81,58 \%$ no tiene un soporte que le permita ajustar la altura.

4. El 32,88\% utiliza mouse periférico y un $67,12 \%$ no lo usa.

5. Un $21,27 \%$ cuenta con un teclado periférico y un $78,73 \%$ no posee.

En la tabla 1 se muestra el porcentaje de quienes han presentado molestias y la intensidad, donde 1 es sin molestias, a 5 molestias muy fuertes. 
Tabla 1. Prevalencia de molestias por región corporal según intensidad.

\begin{tabular}{|l|c|c|c|c|c|}
\hline \multirow{2}{*}{$\begin{array}{l}\text { Región } \\
\text { corporal }\end{array}$} & $\mathbf{1}$ & $\mathbf{2}$ & $\mathbf{3}$ & $\mathbf{4}$ & $\mathbf{5}$ \\
\cline { 2 - 6 } & $19,13 \%$ & $22,02 \%$ & $28,52 \%$ & $16,97 \%$ & $13,36 \%$ \\
\hline Cuello & $31,77 \%$ & $24,55 \%$ & $23,83 \%$ & $11,91 \%$ & $7,94 \%$ \\
\hline Hombro & $11,55 \%$ & $16,61 \%$ & $29,24 \%$ & $21,30 \%$ & $21,30 \%$ \\
\hline Espalda & $52,71 \%$ & $23,47 \%$ & $17,33 \%$ & $3,97 \%$ & $2,53 \%$ \\
\hline $\begin{array}{l}\text { Codo/ } \\
\text { antebrazo }\end{array}$ & $40,43 \%$ & $26,35 \%$ & $19,49 \%$ & $9,03 \%$ & $4,69 \%$ \\
\hline $\begin{array}{l}\text { Mano/ } \\
\text { muñeca }\end{array}$ & $31,05 \%$ & $25,27 \%$ & $24,55 \%$ & $10,83 \%$ & $8,30 \%$ \\
\hline $\begin{array}{l}\text { Piernas/ } \\
\text { rodillas }\end{array}$ & & & & & \\
\hline
\end{tabular}

\section{Aspectos del ambiente}

1. El 54,15\% cuenta con un lugar de trabajo destinado al espacio laboral, y el $45,85 \%$ no cuenta con un lugar fijo.

2. El 41,52\% realiza sus actividades en el comedor, el $7,58 \%$ en la cocina, el $57,04 \%$ en la recámara, el 23,83\% en la estancia, el 7,94\% en una oficina, y el 1,81\% restante en otro lugar.

3. El $76,17 \%$ considera que su familia respeta su espacio y tiempo de trabajo, y el $23,83 \%$ restante no lo considera así.

4. El 38,63\% percibe su entorno de teletrabajo como ruidoso, y el $61,37 \%$ restante no lo considera así.

5. De las personas que consideran su entorno de trabajo como ruidoso, al 23,61\% le genera molestia, al 37,96\% no le permite concentrarse, y al 38,43\% dificulta la comunicación con colegas y/o alumnos.

6. Cuando se concentran, el 16,97\% usa audífonos con música, un 10,11\% utiliza audífonos sin música, un $0,72 \%$ usa tapones o sistema similar, un 21,30\% escucha música ambiental de fondo, y un 50,90\% no realiza ninguna de las actividades mencionadas anteriormente.

7. A un $14,08 \%$ la iluminación no le permite realizar el teletrabajo sin generar alguna molestia o inconveniente, y al 85,92\% restante no le genera ningún problema.

8. Los problemas que generan que la iluminación sea inadecuada (si aplica), un $36,79 \%$ es por la iluminación escasa, un 37,31\% es por reflejos y/o resplandores molestos y un 25,91\% es por problemas de contraste.

9. Un $88,09 \%$ refiere que la temperatura ambiental es adecuada, y un 11,91 \% considera que no lo es.

Aspectos de carga mental, exigencias laborales y horarios de trabajo

1. Un $14,44 \%$ considera que tiene $100 \%$ control sobre el ritmo de trabajo, un 53,07\% el $75 \%$ del tiempo, un $23,83 \%$ el $50 \%$ del tiempo, un $6,50 \%$ solo el $25 \%$ del tiempo, y un $2,17 \%$ considera que nunca tiene el control. 
2. En cuanto a la supervisión durante la jornada laboral, un $7,22 \%$ lo tiene el $100 \%$ del tiempo, un 18,05\% el 75\% del tiempo, un 24,55\% el 50\% del tiempo, un 25,63\% el $25 \%$ del tiempo, y un 24,55\% nunca tiene supervisión.

3. El 19,49\% puede negociar los plazos y entrega de resultados el 100\% del tiempo, un 31,41\% el $75 \%$ del tiempo, un $23,10 \%$ el 50\% del tiempo, un $12,64 \%$ el $25 \%$ del tiempo, y un $13,36 \%$ nunca.

4. $\mathrm{Al} 3,97 \%$ las exigencias temporales sobrepasan sus capacidades para manejarlas el $100 \%$ del tiempo, al 18,41\% el 75\% del tiempo, al 24,91\% el 50\% del tiempo, al 22,74\% el 25\% del tiempo, y al 29,96\% nunca.

5. Un 61,01\% tiene establecidos horarios de trabajo y descanso, un 31,05\% no los tiene, y un $7,94 \%$ prefiere no contestar.

6. Un $55,23 \%$ respeta el horario de trabajo, y un $44,77 \%$ no lo hace.

7. Del total de la población que no le es posible respetar los horarios de trabajo, los principales motivos son, las tareas domésticas en un 32,13\%, porque la jefatura y/o colegas no respetan el horario de trabajo en un $21,66 \%$, porque los alumnos no respetan el horario en un 44,04\% y, por último, un 34,30 \% no ha tenido problemáticas de este tipo.

8. Un $84,48 \%$ tiene un horario entre 8:00 y 12:00 horas, un 43,68\% entre 12:00 y 16:00 horas, un 38,27\% entre 16:00 y 20:00 horas, un 14,80\% entre 20:00 y 24:00 horas, y un 3,97\% entre 24:00 y 4:00 horas.

9. En un $80,14 \%$ se respetan las tareas domésticas y responsabilidades sobre los niños y/o adultos que requieren ayuda en el hogar, en un $18,77 \%$ no se respeta, y en el 1,08\% no aplica o difiere de las dos respuestas anteriores.

10. Las actividades domésticas que más distraen durante el desarrollo del teletrabajo son, en un $55,23 \%$ cocinar, en un 56,32\% el aseo, en un $31,05 \%$ las compras de insumos, en un $20,58 \%$ el lavado de ropa, un $29,96 \%$ el cuidado de niños y/o adultos que lo requieren, y un 29,60\% el apoyo a hijos en actividades escolares.

\section{Teletrabajo y consecuencias}

A continuación, en la tabla 2, se presentan los porcentajes de respuesta respecto a la percepción en cuanto a si han mejorado o empeorado en los aspectos mencionados. 
Tabla 2. Nivel de percepción del teletrabajo y sus consecuencias.

\begin{tabular}{|c|c|c|c|c|c|}
\hline \multirow[b]{2}{*}{ Aspectos } & \multicolumn{5}{|c|}{ Escala de percepción } \\
\hline & $\begin{array}{c}\text { Ha } \\
\text { mejorado }\end{array}$ & $\begin{array}{c}\text { Se mantiene } \\
\text { igual }\end{array}$ & $\begin{array}{c}\text { Ha } \\
\text { empeorado }\end{array}$ & No aplica & $\begin{array}{c}\text { Prefiero } \\
\text { no } \\
\text { contestar }\end{array}$ \\
\hline Salud & $11,91 \%$ & $65,70 \%$ & $18,41 \%$ & $2,17 \%$ & $1,81 \%$ \\
\hline $\begin{array}{l}\text { Productividad } \\
\text { laboral }\end{array}$ & $13,00 \%$ & $58,84 \%$ & $23,10 \%$ & $2,17 \%$ & $2,89 \%$ \\
\hline $\begin{array}{l}\text { Calidad del } \\
\text { trabajo }\end{array}$ & $14,80 \%$ & $59,21 \%$ & $22,74 \%$ & $1,08 \%$ & $2,17 \%$ \\
\hline $\begin{array}{l}\text { Relaciones con } \\
\text { colegas }\end{array}$ & $9,39 \%$ & $64,62 \%$ & $19,86 \%$ & $4,69 \%$ & $1,44 \%$ \\
\hline $\begin{array}{l}\text { Relaciones con } \\
\text { jefatura }\end{array}$ & $8,66 \%$ & $74,01 \%$ & $12,27 \%$ & $3,97 \%$ & $1,08 \%$ \\
\hline $\begin{array}{l}\text { Relaciones con } \\
\text { clientes/alumnos }\end{array}$ & $11,91 \%$ & $53,79 \%$ & $28,16 \%$ & $3,97 \%$ & $2,17 \%$ \\
\hline $\begin{array}{l}\text { Relaciones con } \\
\text { su familia }\end{array}$ & $31,41 \%$ & $55,23 \%$ & $12,27 \%$ & $0,36 \%$ & $0,72 \%$ \\
\hline
\end{tabular}

1. Un 11,19\% está preocupado de que lo despidan, no le renueven el contrato, o temen perder sus clientes, un $16,97 \%$ le preocupa que cambien las labores que realiza contra su voluntad, un $19,49 \%$ siente que su jefatura/clientes reconocen sus labores, y un 52,35\% puede responder adecuadamente a las demandas laborales y domésticas.

2. En una escala de 1 a 5, donde 1 es malo y 5 es excelente, un 11,55\% evaluó su experiencia desarrollando teletrabajo en 5 , un $48,01 \%$ en 4 , un $32,85 \%$ en 3 , un $5,42 \%$ en 2 , y un $2,17 \%$ en 1 , como se muestra en la figura 2.

Figura 2. Percepción de la experiencia desarrollando teletrabajo.

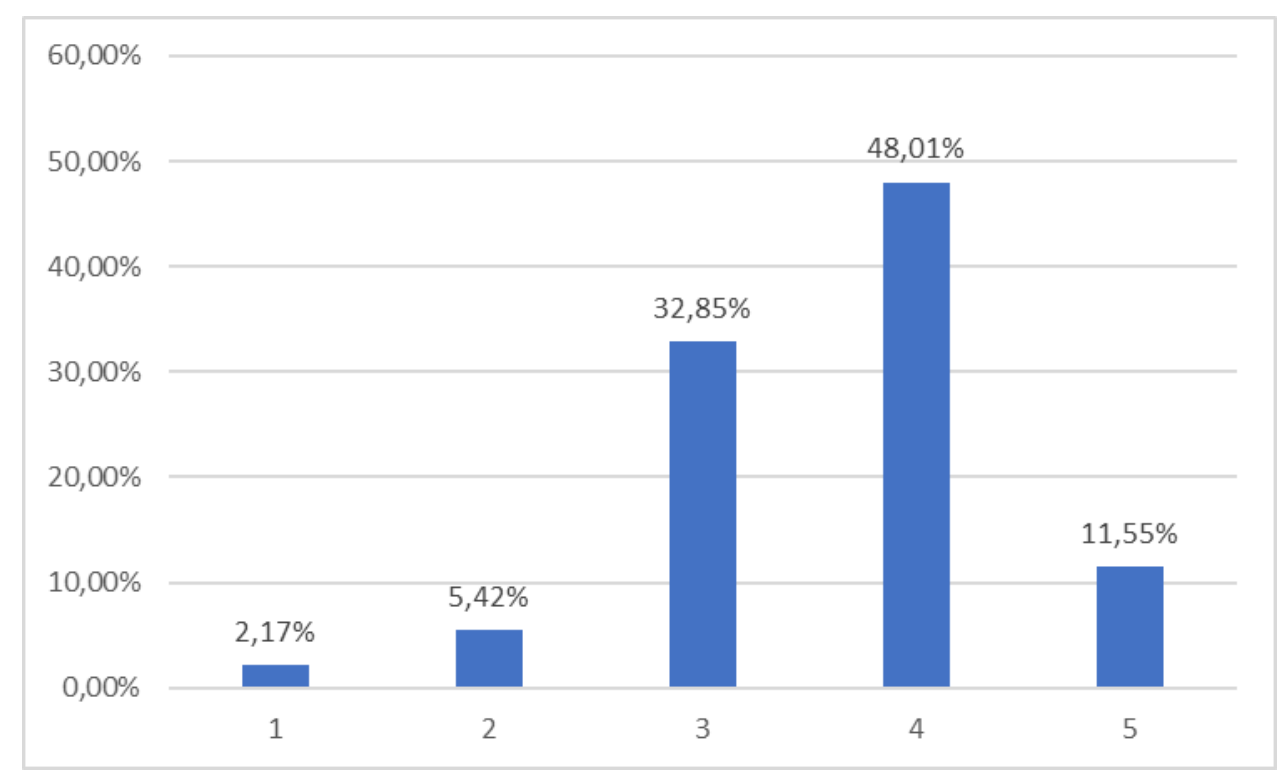




\section{Discusión}

Antes de la pandemia, el 84\% no había desarrollado teletrabajo, y aun así, un 79,42\% de los participantes, no recibió entrenamiento y/o capacitación en ergonomía ni las instrucciones de cómo realizar las actividades. Lo anterior expone a riesgos de daños a la salud, como el aumento de peso, molestias físicas y una alimentación menos saludable. En comparación con el estudio de referencia un 67,2\% no había desarrollado teletrabajo y un 70\% no recibió instrucciones en teletrabajo.

Una de las problemáticas más notorias es que casi el 50\% no cuenta con un lugar de trabajo fijo y el 14,08\% tiene problemas de iluminación, un inconveniente que puede generar molestias y daños a la vista al pasar el tiempo. En cuanto a malestares físicos, destacan el dolor en el cuello y espalda, pues el 13,36\% y 21,30\% dice que ha presentado molestias muy fuertes, por ejemplo, más del 50\% de la población sufre de alguna molestia física, y se relaciona al hecho de que, el porcentaje que considera las posturas al trabajar es menor a los que no lo hacen; solamente en la espalda el porcentaje de $35 \%$ es mayor en quienes no consideran su postura de esta parte del cuerpo. En comparación con el estudio de la Universidad de Concepción y la SOCHERGO, las áreas en que los participantes refieren mayor porcentaje de molestias son: cuello y hombros (49,4\%), espalda (49,1\%), codos, antebrazos y muñecas $(28,2 \%)$, cadera $(18,7 \%)$ y piernas, rodillas y pies $(18 \%)$.

El 45\% no cuenta con un lugar fijo para realizar el trabajo, hecho que dificulta que las condiciones sean adecuadas para realizar el teletrabajo, pues se tendrían que diseñar y adaptar varios espacios en el hogar. El 31\% no cuenta con un horario de trabajo, y el $44 \%$ no cumple con el horario establecido, lo que significa que un porcentaje de las personas que tienen planeado el tiempo de trabajo, no les es posible seguir con lo que planificaron inicialmente.

Estos elementos encontrados coinciden con el análisis realizado por Sánchez-Toledo y Agustín (2021) sobre los principales efectos del teletrabajo, encontrando que, los efectos positivos identificados con mayor frecuencia son: 1) mayor productividad, ante la ausencia de interrupciones, 2) aumento de la satisfacción laboral del trabajador y reducción de estrés, 3) mayor flexibilidad en los horarios, que deriva en una mayor cantidad de tiempo para dedicar a actividades de la vida personal, y 4) aumento de la percepción de un mayor control sobre la gestión del tiempo y las actividades laborales cotidianas.

Por otra parte, los efectos negativos más frecuentes son: 1) problemas asociados a la falta de infraestructura tecnológica adecuada para realizar las labores cotidianas, lo cual genera estrés en el trabajador al consumir tiempo de la jornada de trabajo, 2) extensión de la jornada laboral y empalme de las labores cotidianas con los deberes del trabajo, 3) sensación de falta de apoyo organizacional a la hora de realizar labores complejas o nuevas, 4) sensación de soledad y falta de cohesión del teletrabajador, y 5) retroalimentación irregular por parte de los supervisores. 


\section{Conclusiones}

Como se mencionó antes, el teletrabajo es la acción de llevar a cabo las tareas designadas por un jefe desde un lugar fuera de las instalaciones de la empresa o lugar establecido, haciendo uso de las tecnologías de la información y la comunicación. Para identificar la experiencia de los teletrabajadores en el trabajo a distancia, es necesario conocer su opinión en distintos puntos. En este caso, con ayuda de un cuestionario sobre el teletrabajo, fue posible recabar información de 277 personas por medio de este instrumento de 50 preguntas sobre el lugar del trabajo.

En cuanto a la experiencia personal, la mayoría calificó la experiencia como buena o regular, solo un porcentaje pequeño, entre el $1 \%$ y $5 \%$ aproximadamente, calificó la realización del trabajo a distancia, de excelente y mala respectivamente. Lo anterior indica de manera general su percepción, pero, es posible identificar más a fondo las condiciones ergonómicas. Por ejemplo, el 49\% no realiza actividad física de manera regular, el $71 \%$ realiza menos actividad física que cuando trabajaba de manera presencial, el 33\% no hizo ajustes a su alimentación, y un 44\% aumentó de peso. Estos datos se relacionan entre sí, y genera atención en los altos porcentajes, pues los cuatro puntos son importantes para la salud de la población.

Las actividades que presentan mayor riesgo de acuerdo al nivel de manifestación, es que el $81 \%$ no tiene un soporte para adaptar la altura del monitor, $78 \%$ no cuenta con teclado periférico, $71 \%$ realiza menor actividad física que cuando trabajaban presencialmente, un poco más de la mitad considera que tiene control aproximadamente el $75 \%$ del tiempo en sus tareas, la mayoría considera que las relaciones laborales y la productividad se ha mantenido igual, el porcentaje que cree que ha empeorado es mayor al que considera que ha mejorado y solamente en las relaciones familiares se observa un porcentaje mayor de mejora, el 38,6\% considera su espacio de trabajo como ruidoso, el 23,8\% considera que su familia no respeta su espacio, el 18,7\% dice que no se respetan las tareas domésticas, 16,9\% teme que cambien las labores que realiza sin previo aviso, 14,08\% tiene molestias por la iluminación y el 3,97\% realiza su trabajo de las 24:00 a 4:00.

\section{Recomendaciones}

La recomendación para el presente estudio es que se capten más respuestas con el fin de lograr un nivel de confianza mayor. Además, al ser el teletrabajo una modalidad que ha ido ganando mayor relevancia, se pueden generar más líneas de investigación que permita complementar la presente, por ejemplo, la evaluación económica de home office en la población docente, definir el costo beneficio de mejorar las condiciones ergonómicas del trabajo, y evaluaciones con otro tipo de metodologías ergonómicas. Esas temáticas y, muchas otras, ayudarían a crear una base sólida de investigación que facilite la adopción de medidas que disminuyan los riesgos ergonómicos propios al teletrabajo.

Por último, se recomienda efectuar seguimientos de los teletrabajadores para comprobar el grado de evolución, considerando los diversos impactos que la modalidad puede tener sobre la persona, a nivel profesional, personal, familiar y psíquico. 


\section{Referencias}

Álvarez, P. (2018, octubre). Teletrabajo en la experiencia extranjera. Los casos de Argentina, Brasil, Bélgica, Estados Unidos de Norteamérica, Francia, Italia y Portugal. Asesoría Técnica Parlamentaria. SUP. No. 117212. Biblioteca del Congreso Nacional de Chile. https://obtienearchivo.bcn.cl/obtienearchivo?id=repositorio/10221/25913/2/PA Teletra bajo 2018.pdf

Barona-Betancourt, R. (2013). Actualidad del teletrabajo en Colombia. Revista Internacional y Comparada de Relaciones Laborales y Derecho del Empleo, 1(3),118-125.

Cifre-Gallego, E., \& Alonso-Fabregat, M., (2002). Teletrabajo y salud: Un nuevo reto para la $\begin{array}{llll}\text { psicología. Papeles del Psicólogo, } & \text { (83),55-61. }\end{array}$ https://www.redalyc.org/articulo.oa?id=778/77808308

Fernández-Tapia, J., \& Bravo-Salazar, R. G., (2019). El teletrabajo en América Latina: Derecho de segunda y cuarta generación y de ciudadanía digital. Revista de conflictos sociales latinoamericanos, (9), 17-38. https://www.criticayresistencias.com.ar/revista/article/view/118

Martínez, M., (2020). Diputados avalan dictamen que regula home office. El Economista. https://www.eleconomista.com.mx/empresas/Diputados-avalan-dictamen-que-regulahome-office-20200721-0011.html

Miranda, F. (2020, 19 de marzo). Sólo 2 de cada 10 empresas listas para "home office" en México ante Covid-19: UNAM. Grupo Milenio. https://www.milenio.com/politica/comunidad/home-office-mexico-2-10-empresasunam

Mondelo, P., Gregori-Torada, E., De Pedro-González, O., \& Gómez-Fernández, M., (2013). Ergonomía 4. El trabajo en oficinas. Edicions UPC. https://upcommons.upc.edu/bitstream/handle/2099.3/36777/9788476539828.pdf

Organización Internacional del Trabajo. (2017). CDMX lidera el tema del teletrabajo en México. http://www.oit.org/mexico/noticias/WCMS_561787/lang--es/index.htm

Osio-Havriluk, L. (2015). Salud y seguridad en el teletrabajo. Caso: Argentina. Visión Gerencial, (2),410-426. https://www.redalyc.org/articulo.oa?id=4655/465545899009

Rodríguez-Mejía., M. (2007). El teletrabajo en el mundo y Colombia. Gaceta Laboral, 13(1), 29-42. $\quad$ http://ve.scielo.org/scielo.php?script=sci $\operatorname{arttext\& pid=S1315-}$ 85972007000100002\&lng=es\&tlng=es

Rodríguez-Moreno, D. C. (2017). Legislación laboral para el teletrabajo. Revista Principia Iuris, 14(27), 166-183.

http://revistas.ustatunja.edu.co/index.php/piuris/article/view/1406

Sánchez-Toledo, L., \& Agustín, M. (2021). Efectos del teletrabajo sobre el bienestar de los trabajadores. Revista de la Asociación Española de Especialistas en Medicina del Trabajo, 3o(2), 234-254. http://scielo.isciii.es/scielo.php?script=sci $\operatorname{arttext\& pid=S1132-}$ $\underline{62552021000200234 \& \operatorname{lng}=\mathrm{es} \& \ln \mathrm{l}=\mathrm{es}}$

Secretaría de Gobernación. (2019, 2 de julio). Ley federal del trabajo. Diario Oficial de la Federación.

https://www.dof.gob.mx/nota detalle.php?codigo $=5564651 \&$ fecha $=02 / 07 / 2019$

Silva-Ambríz, A., García-Flores, J., \& Fuentes-Rojas, J., (2013). Carencia de un marco legal regulatorio del teletrabajo en México. Letras jurídicas, Revista Multidisciplinar del CEDEGS, 14(28), 109-120. https://www.uv.mx/cedegs/files/2020/10/Revista-LetrasJuridicas-numero028.pdf 
Universidad de Concepción y Sociedad Chilena de Ergonomía. (2020). Entorno ergonómico del teletrabajo en situación de pandemia. https://www.sochergo.cl/wpcontent/uploads/2020/o8/ERGONOMIA-Y-TELETRABAJO-UDEC-SOCHERGO2020.pdf

Valdivieso-Taborga, C., Valdivieso-Castellón, R., \& Valdivieso-Taborga, O. (2011). Determinación del tamaño muestral mediante el uso de árboles de decisión sample size determination using decision trees. Revista Investigación \& Desarrollo, 1(11), 53-80. https://doi.org/10.23881/idupbo.011.1-4e

Valle-Mendoza, G., (2018). Teletrabajo, necesidad y solución en Méjico. Un estudio exploratorio de sus barreras culturales y obstáculos. Revista Latinoamericana de Investigación en Organizaciones, Ambiente y Sociedad, 9(12), 153-176. https://doi.org/10.33571/teuken.vgn12a7 